\title{
The measurement of the reflection and transmission properties of conducting fabrics to milli-metric wave frequencies
}

\author{
C. H. Oxley ${ }^{*}$, J. Williams ${ }^{*}$ R. Hopper ${ }^{*}$, H. Flora ${ }^{*}$, D. Eibeck ${ }^{*}$ and C. Alabaster ${ }^{++}$ \\ *De Montfort University \\ ${ }^{++}$Cranfield University (DCMT Shrivenham)
}

\begin{abstract}
There is increasing interest in conducting fabrics and their uses at RF and microwave frequencies. This paper for the first time looks at the reflection and transmission measurements of bobbinet and knitted materials from around $8 \mathrm{GHz}$ into the millimetric frequency range $110 \mathrm{GHz}$, where the material geometry is comparable to the wavelength of the wave. Bobbinet materials were found to behave like lossy dielectrics and may be useful in the construction of thin light-weight screening and absorption planes. While the knitted materials, with very small mesh geometry, gave a reflection coefficient which was comparable to a metal foil.
\end{abstract}

\section{Introduction}

Interest in conducting fabrics has significantly increased over the last decade particularly seeing the integration of electronic circuitry for example key-pad and light emitting diodes to provide visual impact within the garment [1]. It is also expected that nano-technologies will enable integration of arrays of transistors, diodes and other electronic components within the weave of the material, greatly enhancing capability of these garments $[2,3]$. The technology has also shown to provide electromagnetic shielding by reflection and absorption of radio frequencies (RF) and microwave wave signals making it an ideal light weight material for screening and reflecting electromagnetic (EM) energy. Applications in this area have already been exploited, for example navel decoys, screening of the human body from RF energy [4] and antennas [5]. The potential of using the material in thin EM shielding absorption screens, compared with the $\lambda / 4$ thickness associated with Salisbury screens, for applications in radar tracking, wireless communication and electronic warfare has been shown in the frequency range of 3 to $6 \mathrm{GHz}$ [6]. However, very little work appears to have been published regarding the $\mathrm{RF} / \mathrm{microwave}$ characterisation of conducting fabrics and in particular into the milli-metric frequency band (30 to 
$300 \mathrm{GHz}$ ) where the fibre spacing can be of a similar order to the signal wavelength. The microwave measurement of the material with knowledge of its structure may lead to the design of enhanced reflection/absorption qualities for example thin and flexible highly absorbing fabrics over wide bandwidths and ultimately with adaptive properties. These materials may well have many applications in both the commercial and the military sectors of the market.

Conducting fabrics are easy and cheap to manufacture and consist of conducting yarns woven into a fabric using the standard weaving or knitting manufacturing processes developed over the last couple of centuries in the fabric industry [7, 8]. Examples, of different methods of manufacturing fabric are shown in Figure 1. In bobbinet the vertical yarn is the warp, the diagonal yarns form hexagons. In woven fabrics the vertical yarn is again the warp whilst the horizontal yarns are the weft. In knitted fabrics the structures are slightly different, weft knits have horizontal yarns forming loops named a course with the vertical column of loops known as a wale. Warp knits are characterised by loops substantially along the length of the fabric.

It seemed reasonable to assume that materials with different weave geometry and yarn material will have significantly different $\mathrm{RF} /$ microwave properties and this is experimentally explored within this paper. A number of commercially available conducting fabrics have been characterised by measuring the small-signal reflection $\left(\mathrm{s}_{11}\right)$ and transmission $\left(\mathrm{s}_{21}\right)$ characteristics over an extended microwave frequency range of 9 to $110 \mathrm{GHz}$. The chosen fabrics had a range of mesh geometries and yarn materials and in particular, the bobbinet materials had hexagonal mesh dimensions which are comparable to the wavelength at $100 \mathrm{GHz}$. However, at the lower frequencies (X-band) the wavelength $(30 \mathrm{~mm}$ at $10 \mathrm{GHz})$ is much greater than the mesh geometry of all the tested materials. The materials were also rotated in order to ascertain whether they exhibited polarisation effects. Further, some comparative tests with thin aluminium foil were conducted at the lower frequency end of the measurement spectrum. A number of interesting features were identified and are briefly discussed.

\section{Microwave Characterisation}

The microwave frequency range 8 to $110 \mathrm{GHz}$ is very wide and to encompass the bandwidth two methods of characterisation were used and at two different 
organisations. At X-band ( 8.0 to $12.4 \mathrm{GHz})$ a waveguide transmission method was used, while at the higher frequencies $(50$ to $110 \mathrm{GHz})$ a free space method was used. The waveguide transmission method was developed to enable the comparison of small area sections of materials in the laboratory and it could be extended at a later date to include different environmental conditions, for example, at elevated temperature and pressure. This approach also enabled good contact impedance with the fabric across the rectangular waveguide section, which would be more difficult in a transmission line with a centre conductor [9]. Therefore, the measurement of the scattering parameters will be directly related to the introduction of the fabric into the waveguide test-fixture. Note, in using this method the wave impedance is a function of frequency unlike the free space method. The waveguide test fixture consisted of identical input and output waveguide sections (WR90) with coaxial transitions to connect into the network analyser (Figure 2). A small sample of the conducting fabric could be placed between the waveguide input and output sections. The reflection $\left(\mathrm{s}_{11}\right)$ and transmission $\left(\mathrm{s}_{21}\right)$ s-parameters were measured using an Anritsu 337369A automatic vector network analyser (VNA). The standard waveguide calibration technique was used which consists of two accurately known lengths of waveguide and a waveguide offset short circuit. The calibration of the measurement system was checked with no material between waveguide sections and a metal plate. With a metal plate $s_{11}=$ approached 1 (1.01 to 0.99$)$ and $s_{21}$ approached 0 (-66 to $\left.-80 \mathrm{~dB}\right)$ between 8 to $11 \mathrm{GHz}$. This frequency range was used for the reflection/transmission measurements. The fabric sample orientation was changed ( 0 and 90 degrees respectively) during the measurements and three samples showed little or no polarisation of the incident wave. However, one sample (A) consisting of a longitude yarn (warp) which was conducting and lateral yarn (weft) which was non-conducting did show polarization effects. The waveguide method also enabled a comparative measurement of the reflection coefficient and absorption between conducting fabrics and aluminium foil, albeit over a very limited frequency range.

The high frequency measurement was made in two bands 50 to $65 \mathrm{GHz}$ and 74 to 110 GHz. The reflection and transmission coefficients of the fabric were measured, using a free space measurement technique and an Anritsu ME7808B vector network analyser. The measurements were undertaken by Cranfield University, Shrivenham, UK. The measurement method required cutting a circular sample of approximately 
$100 \mathrm{~mm}$ diameter from the conducting fabric and placing it in a frame which was positioned mid-way between a pair of standard gain waveguide horns (Figure 3). All the measurements were performed using a vertical plane of polarization $\left(0^{\circ}\right)$ and the reflection $\left(\mathrm{s}_{11}\right)$ and transmission $\left(\mathrm{s}_{21}\right)$ s-parameters were measured. Measurements were also made for sample orientations of $0^{\circ}, 45^{\circ}$ and $90^{\circ}$ respectively. To exclude multi-path reflections a time gated frequency domain measurement was adopted. The reflection measurement was referenced to the reflection coefficient of a smooth flat solid metal plate in place of the sample between the horns, and the transmission measurement was referenced to free space between the horns. The distance between the horns was sufficient to ensure far-field conditions.

A pair of Flann 25240-20 horns was used for the 50 to $65 \mathrm{GHz}$ measurement and fed into a WR15 waveguide. A horn to sample offset of $160 \mathrm{~mm}$ was chosen to ensure the sample was in the far-field of each horn (given by the threshold distance of $2 \mathrm{D}_{1}{ }^{2} / \lambda$, where $\lambda$ is the wavelength and $D_{1}$ the broadest dimension of the waveguide horn), therefore, the incident signal on the sample can be assumed to approximate to a plane wave. Furthermore, the sample is sufficiently large so as to intercept the whole beam from the horns and so approximates to an infinitely large sample.

For the 74 to $110 \mathrm{GHz}$ band, a pair of Flann 27240-20 horns, were used as receive and transmit heads, respectively. These fed a WR10 waveguide and the sample offset to the far-field threshold was $125 \mathrm{~mm}$. Again the sample intercepts the whole of the beam and therefore approximates to an infinitely large sample.

\section{Fabric Samples}

A number of commercially available conducting fabrics using both bobbinet and knit constructions were chosen for the RF/microwave comparison. These fabrics have been designated as samples A, B, C and D (A \& B bobbinet, C knitted \& D woven) and the measured properties are shown in table1:

The geometry of the four conducting materials was also very different and scan electron microscope (SEM) photographs of the four materials are shown in Figure 4. Sample A contained both a conducting and a non-conducting yarn. Samples A, B, C used silver coated fibres in the conducting yarn, whereas material D the fibres were nickel coated. 
The surface resistance of the material was measured using a dc resistance probe, in which the area between two the flat probes was $5 \mathrm{~cm}^{2}$, this enabled the dc surface conductivity $\sigma$ to be calculated. The effective surface area was estimated by placing a representative area of the fabric on a grid of fine squares. Table 2 gives the sample designation, an estimate of the conductive percentage surface area of the material, and estimated electrical conductivity.

\section{Results and discussion}

Figures 5 and 6 respectively, show the measured reflection $\left|s_{11}\right|$ and transmission $\left|s_{21}\right|$ coefficients between 9 to $110 \mathrm{GHz}$ for the 4 samples. The results for each sample include two angles of orientation ( $0 \& 90$ degrees) to show the maximum variation between reflection and transmission loss measurements. Good repeatability of results was obtained in all the measured frequency bands. At the low frequencies the estimated errors were s $11+/-0.005$ and s $21+/-0.05 \mathrm{~dB}$, and at the milli-metric wave frequencies 57 to $63 \mathrm{GHz}, \mathrm{s} 11+/-0.02, \mathrm{~s} 21+/-0.47 \mathrm{~dB}$ and 76 to $100 \mathrm{GHz}$ s $11+/-0.047$ and $\mathrm{s} 21+/-0.315 \mathrm{~dB}$.

The transmission loss $\left(\mathrm{s}_{21}\right)$ of all four conducting fabrics decreased with increasing frequency. The fabric samples A \& B which were both bobbinet types of material in which the size of the mesh $(\approx 1 \mathrm{~mm})$ is comparable to the wavelength of the signal in the milli-metric wave (MMW) band, showed the lowest transmission loss and reflection coefficients. These materials appeared to behave similarly to lossy dielectrics, and maybe suitable in the design of light-weight, and flexible highfrequency absorption surfaces. Whereas, materials with a much closer woven mesh $(\mathrm{C}$ and D) behaved differently, showing a high reflection coefficient and high transmission loss across the measured frequency range. In particular, sample D a woven material having a large conducting surface area and a yarn coated with nickel, showed the slowest rate of decreasing transmission loss with frequency. The material, however, had a reduced loss and lower reflection coefficient when compared to sample C. Sample C which was a knitted fabric and gave a very high reflection coefficient $\left(s_{11}\right)$ which was independently observed by both collaborating Institutes using different measurement techniques.

The above effect was explored in a little more detail at the lower frequencies. The waveguide measurement system was re-calibrated taking particular care with 
cleanliness and positioning of all the mating sections and calibration parts. The conducting fabrics were re-measured and directly compared with aluminium metal foil. Fabric $\mathrm{C}$ showed almost identical reflection coefficient when compared with the metal foil (Figure 7). The measurements indicate that the very flexible, light weight and high conducting knitted fabrics have very similar reflection coefficient to the less flexible metal foils and over a very wide frequency band (8 to $110 \mathrm{GHz})$.

The waveguide test fixture can be considered as a very low Q cavity. When the conducting fabric is placed in the waveguide cavity the $\mathrm{Q}$ of the structure is changed giving rise to a resonance around $11.4 \mathrm{GHz}$. This property was used to compare to a first order at $11.4 \mathrm{GHz}$ the absorption $(\mathrm{Ab})$ properties of conducting fabrics and the metal foil.

The absorption can be estimated using the following simple expression:

$\mathrm{Ab}=10 \log _{10}\left[1-\left|s_{11}\right|^{2}-\left|s_{21}\right|^{2}\right] \mathrm{dB}$

The measurements indicated that sample A had the highest absorption $(-14 \mathrm{~dB})$, and sample $\mathrm{C}$ the lowest absorption $(-31 \mathrm{~dB})$ closely followed by the metal foil $(-34 \mathrm{~dB})$ as would be expected from a near perfect reflector.

\section{Conclusion}

The experimental results show that conducting fabrics have a number of interesting properties out to milli-metric wave frequencies $(110 \mathrm{GHz})$. The geometry and material of the yarn have, as expected, a direct influence on the microwave properties of the conducting fabric. Bobbinet materials with large mesh geometry appear to behave as lossy dielectrics which may enable their use in the construction of thin, light weight and flexible absorbers. The measurements also show that some fabrics with a very tight mesh and high electrical conductive yarns possess very high reflection coefficients which are comparable to metal foils in X-band.

\section{Acknowledgements}

The work at De Montfort was partially supported by a KTP award on grant 4256 with Swisstulle (UK). Mr. D. Eibeck was the KTP associate.

\section{References}

1) Wilson. A, '100 $21^{\text {st }}$ century textile related innovations' Future materials, issue 
6 (Nov-Dec), 2004, World Textile Publication, Bradford UK.

2) Textiles bring good vibrations to the fight against stress, New Horizons, World Textile Publication, 7/11/02.

3) S. Jung, T. Sturn, C. Lauterbach, G. Stromberg and W. Weber 'Applications of microelectronics and sensors in intelligent textile fabrics' Proc of $12^{\text {th }}$ International Techtextil Symposium, 7 to 10 April, 2003, Frankfurt, Germany.

4) Joyner K.H, Copeland P.R, MacFarlane 'An evaluation of a radio frequency protective suit and electrically conductive fabrics' IEEE trans electromagnetic compatibility, vol 31, issue 2, My 1989, pp 129-137.

5) Pontoppidan K, Vikum H.H, 'Electrical properties of triaxially woven fabrics for reflector antennas' IEEE, antennas and propagation society international symposium, vol 2, June 2003, pp 774-777.

6) S.Simms and V.Fusco 'Thin radar absorber using artificial magnetic ground plane' Electronic Letters, $24^{\text {th }}$ Nov 2005, vol. 41, nos. 24.

7) Kuhn H.N, Child A.D 'Handbook of conducting polymers' $2^{\text {nd }}$ Edition, New York, 1998, pp 993-1013.

8) McIntyre, JE, Daniels, PN, 'Textile terms and Definitions', $10^{\text {th }}$ edition, The Textile Institute 1995

9) Wilson P F, Mark T, J, Adams W, Techniques for Measuring the Electromagnetic Shielding Effectiveness of Materials: Part I:-Far Field Source Simulation, IEEE Transactions on Electromagnetic Compatibility, Vol. 30, No 3, August 1988 


\section{Table Captions}

Table 1, Fabrics tested and metallic yarns

Table 2, Material properties

\section{Figure Captions}

Figure 1, Different methods of manufacturing the conducting fabric

Figure 2, Waveguide transmission measurement

Figure 3, Milli-metric horn transmission measurement

Figure 4, Comparison between the conducting fabric samples using a SEM (40x magnification)

Figure 5, Measured $S_{11}$ from 9 to $110 \mathrm{GHz}$ 
Figure 6, Measured $S_{21}$ from 9 to $110 \mathrm{GHz}$

Figure 7, Comparison of reflection coefficient of bobbinet, weave material and metal foil, between 8 to $12.4 \mathrm{GHz}$. Both fabrics were orientated for maximum reflection.

\begin{tabular}{|l|l|l|l|}
\hline Samples & Yarn diameter (mm) & Yarn material/coating & Geometry \\
\hline $\mathrm{A}$ & 0.1 & $\mathrm{Ag}$ (warp only) & bobbinet \\
\hline $\mathrm{B}$ & 0.125 & $\mathrm{Ag}$ & bobbinet \\
\hline $\mathrm{C}$ & 0.21 & $\mathrm{Ag}$ & warp knit \\
\hline $\mathrm{D}$ & 0.17 & $\mathrm{Ni}$ & woven \\
\hline
\end{tabular}

Table 1 


\begin{tabular}{|l|l|l|}
\hline & material & $\begin{array}{l}\text { conductivity } \\
\text { S/cm }\end{array}$ \\
\hline A & 11 & $9.5 \times 10^{4}$ \\
\hline B & 17 & $2.4 \times 10^{4}$ \\
\hline C & 80 & $1.5 \times 10^{4}$ \\
\hline D & 84 & $0.4 \times 10^{4}$ \\
\hline
\end{tabular}

Table 2

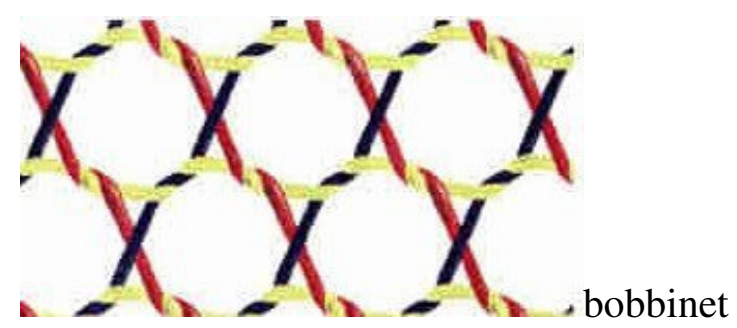




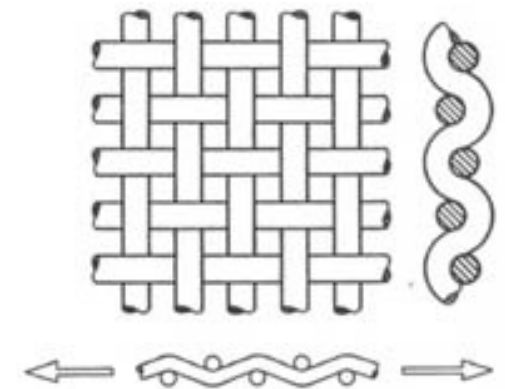

woven $1 x 1$

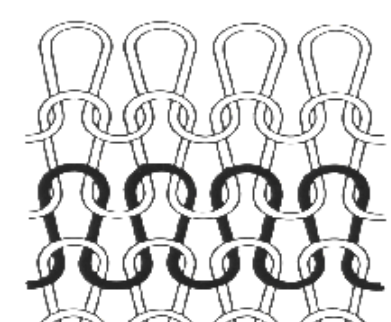

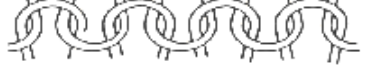

weft knit

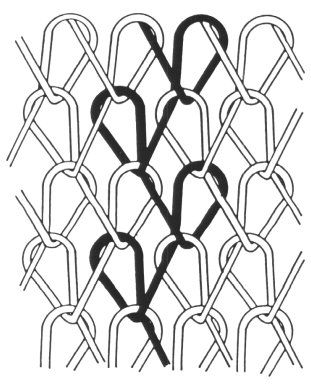

warp knit

Figure 1 


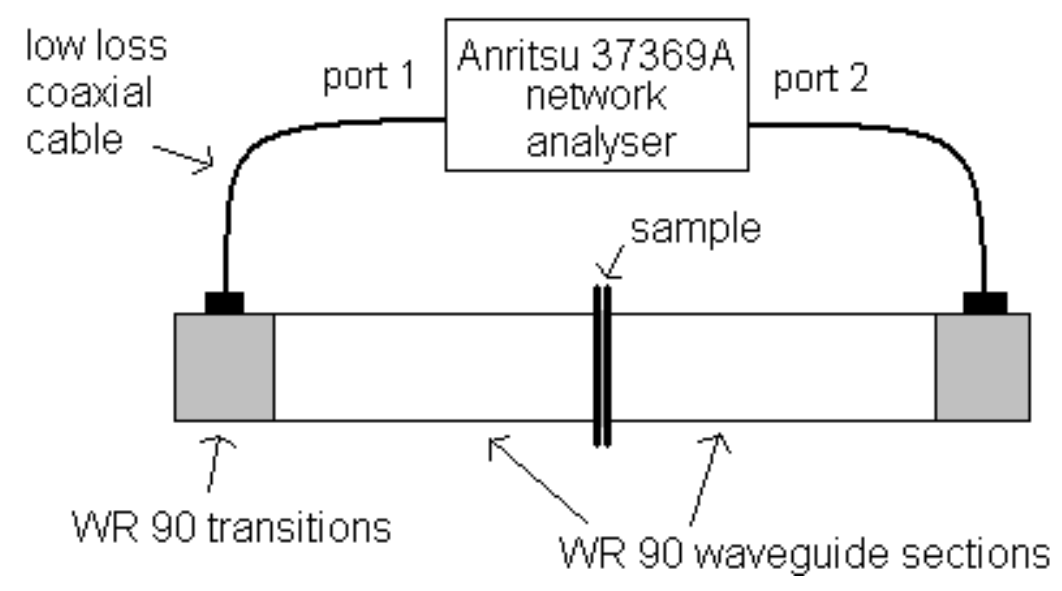

Figure 2 


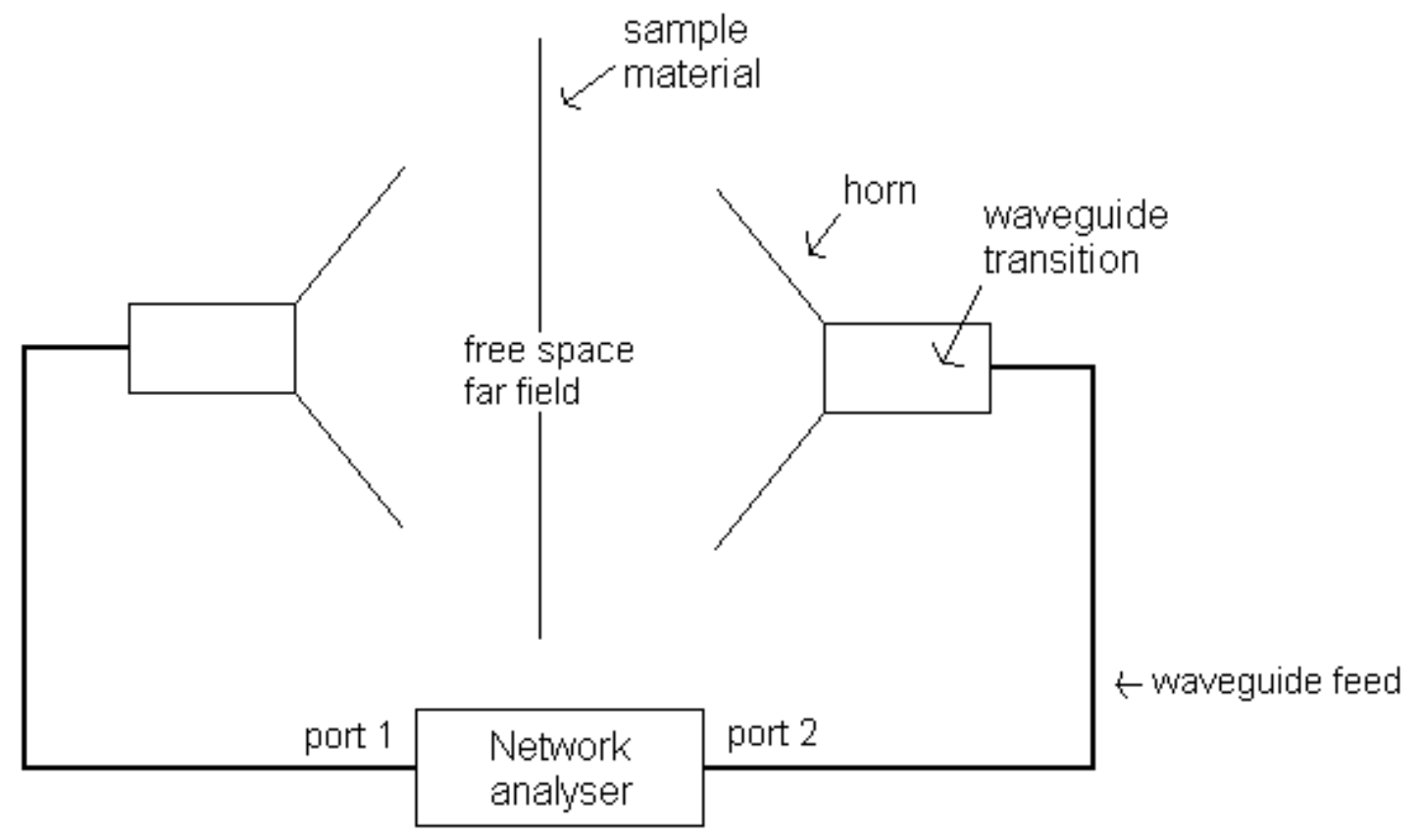

Figure 3 


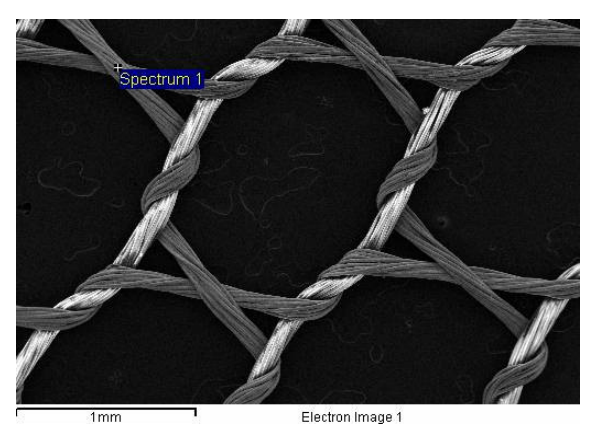

sample A

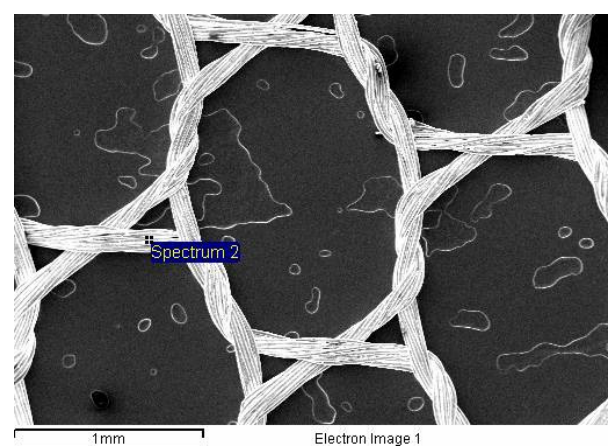

sample B

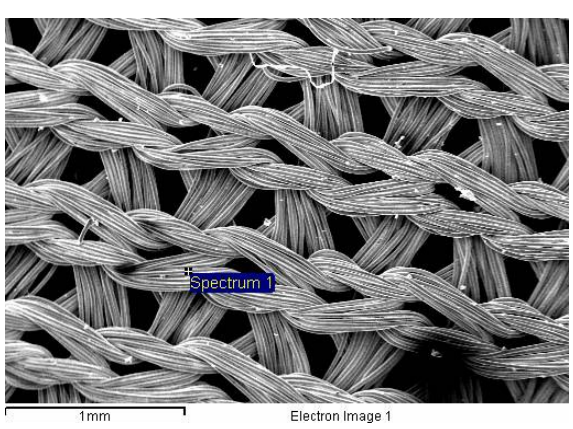

sample C

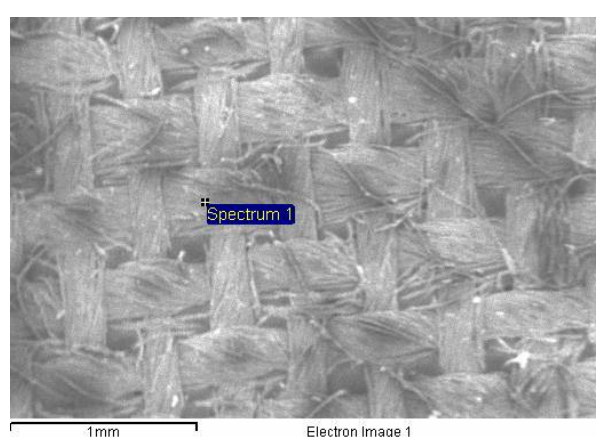

sample D

Figure 2 


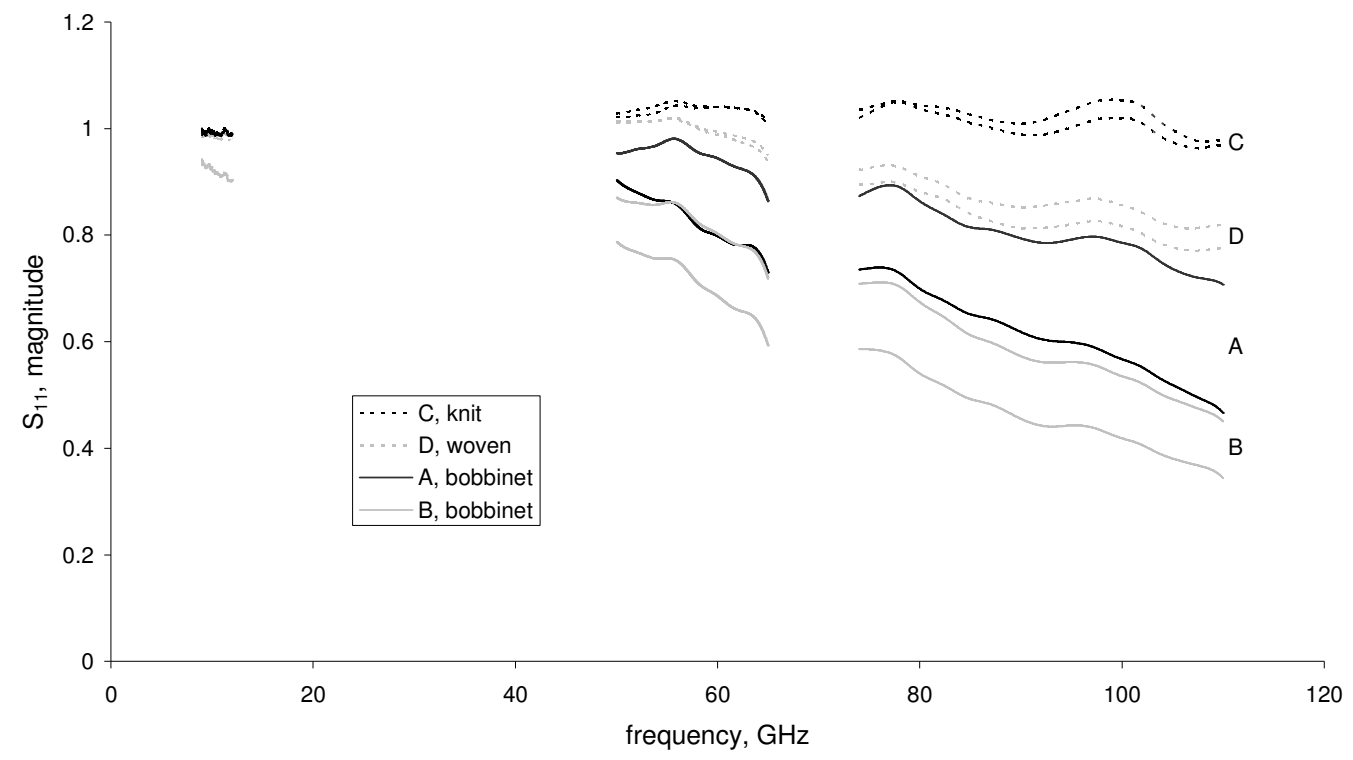

Figure 3 


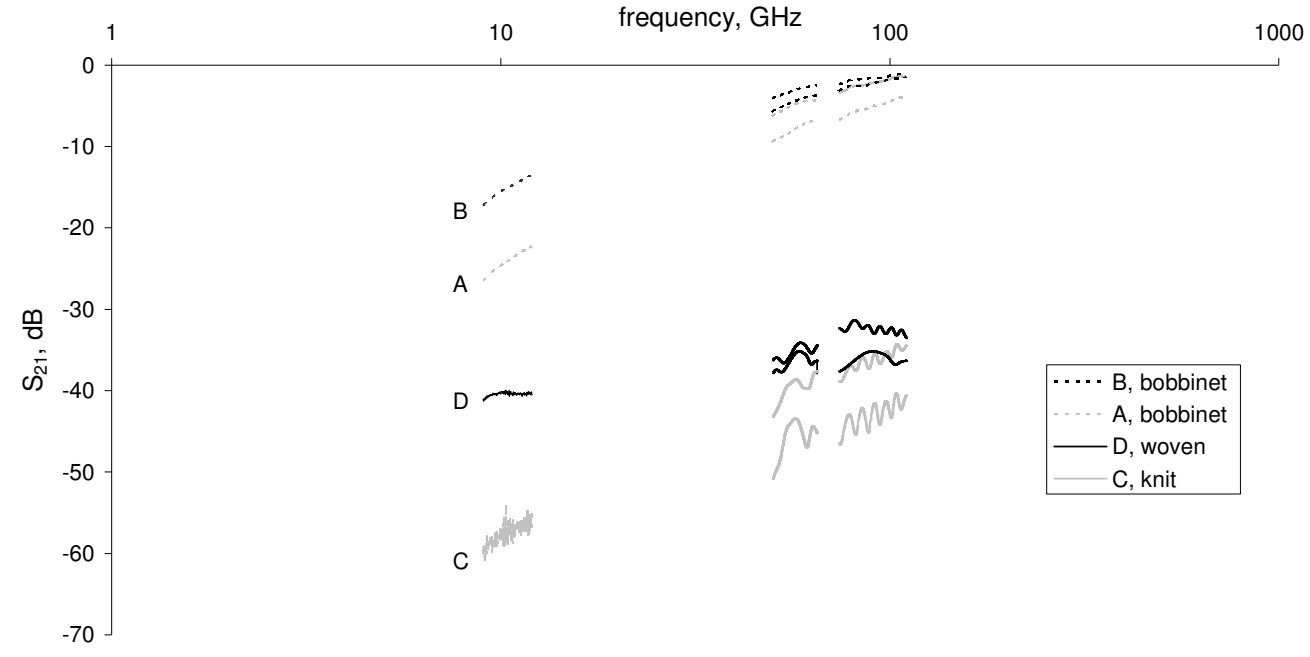

Figure 4 


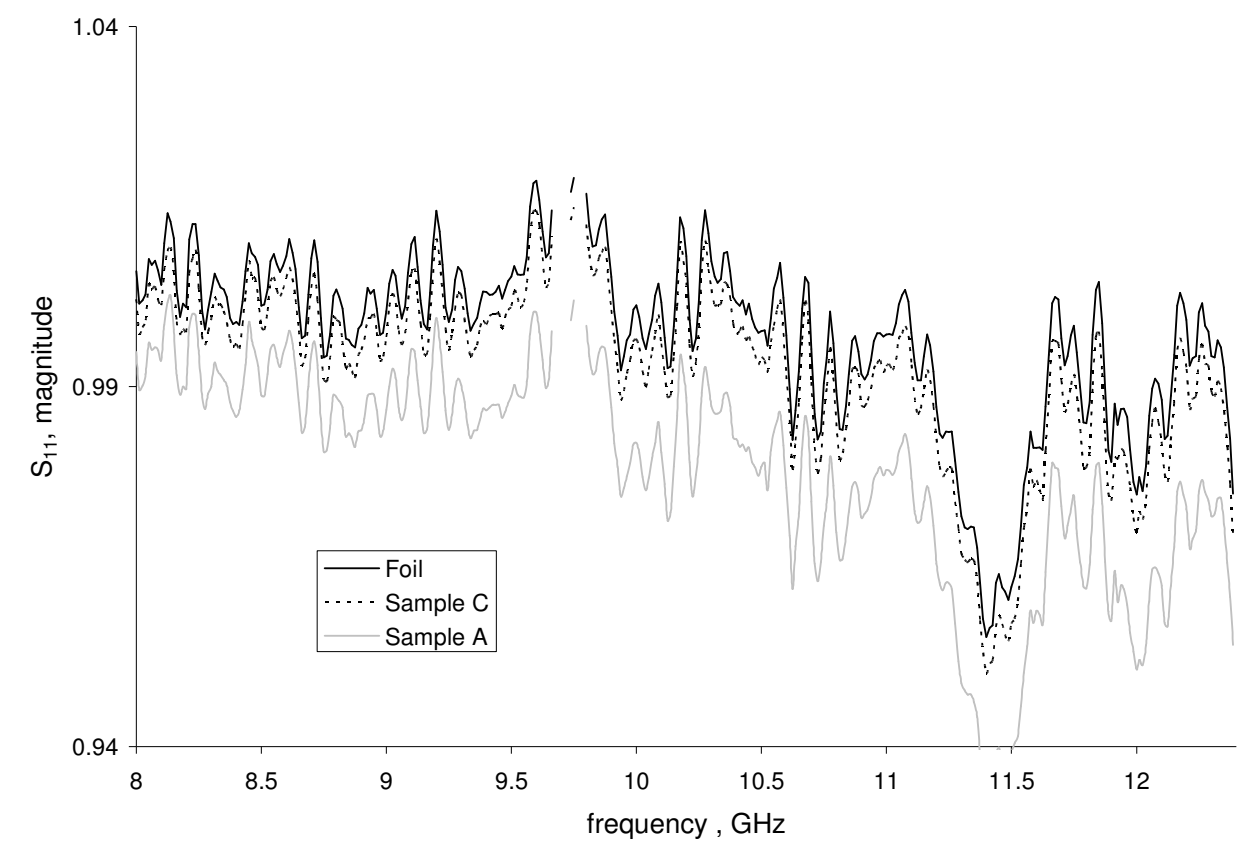

Figure 5 